\title{
Clinical outcomes in primary scalp angiosarcoma
}

\author{
YONG ZHANG, YANWEN YAN, MING ZHU, CHENG CHEN, NANHANG LU, FAZHI QI and JIAQI LIU
}

Department of Plastic Surgery, Zhongshan Hospital, Fudan University, Shanghai 200032, P.R. China

Received November 26, 2018; Accepted June 25, 2019

DOI: $10.3892 / \mathrm{ol} .2019 .10886$

\begin{abstract}
Scalp angiosarcoma is a rare, extremely aggressive cutaneous malignancy with poor patient prognosis. The present study reviewed the cases of 42 patients who presented scalp angiosarcoma and were treated at the Zhongshan Hospital of Fudan University between January 2002 and December 2013. The clinical characteristics, demographics, treatment regimens and outcomes of patients were analyzed, and the overall survival (OS) and recurrence-free survival (RFS) rates were calculated. A total of 42 patients were examined in this study. Surgery was the most common therapeutic measure, and was performed in 39 patients, alone (12 patients), in combination with chemotherapy (14 patients), radiotherapy (6 patients) or 3-modality-therapy (7 patients). The median follow-up time of patients was 28.5 months. The 5-year OS rate was $19 \%$, and the 5-year RFS rate was $10 \%$. Taken together, the results of the present study suggested that patients whose tumor presented a nodular localized lesion had a significantly improved OS rate $(\mathrm{P}=0.0078)$. Patients aged $\geq 70$ years were associated with a lower 5-year OS $(\mathrm{P}=0.0071)$ and $\mathrm{RFS}$ rates $(\mathrm{P}=0.0095)$ vs. patients aged $<70$ years. Different treatments were not identified to be significantly associated with an improved OS or RFS. The present results also indicated that if the tumor presented nodular localized lesions, the patients exhibited a better prognosis than those with a diffuse lesion. Although younger patients had better clinical outcomes, the likelihood of recurrence and mortality remained high for all patients.
\end{abstract}

\section{Introduction}

Cutaneous angiosarcomas are aggressive tumors that arise in either the blood or lymphatic vessels, and account for $\sim 15 \%$ of all head and neck sarcomas and $1 \%$ of all soft tissue sarcomas (1). Cutaneous angiosarcoma has a reported 5-year overall survival (OS) rate between 9 and 30\% (2-4).

Correspondence to: Dr Jiaqi Liu, Department of Plastic Surgery, Zhongshan Hospital, Fudan University, 180 Fenglin Road, Shanghai 200032, P.R. China

E-mail: liujiaqi1213@yahoo.com

Key words: oncology, cutaneous angiosarcoma, scalp, overall survival, recurrence-free survival, surgery
The scalp and face are the most common sites of cutaneous angiosarcoma (4). Previous studies investigated the survival outcomes for cutaneous angiosarcoma of the scalp vs. face, and the results have indicated that scalp angiosarcoma has a worse survival rate compared with facial angiosarcomas $(2,4)$. However, the present study focused on the outcomes of scalp angiosarcoma; therefore, patients with facial angiosarcomas were excluded.

Scalp angiosarcoma has an innocuous presentation $(5,6)$. Complete surgical resection with wide margins is preferred for loco-regional disease (7). Chemotherapy is widely used in patients with lesions that cannot be entirely resected, or who have distant metastatic disease (7). However, the optimal treatment for patients with scalp angiosarcoma has not yet been defined.

Due to its low incidence, the available literature on scalp angiosarcoma primarily consists of case reports, and few large single institution series on scalp angiosarcoma have been published $(5,6,8)$. In the present analysis, a relatively large cohort study was performed, where 42 patients with scalp angiosarcoma were diagnosed and treated at a monocenter (Zhongshan Hospital, Fudan University) over a period of 12 years. The present study analyzed patient and tumor characteristics, as well as treatment algorithms. The potential associations between these parameters and patient outcomes were investigated.

\section{Materials and methods}

Ethics approval and consent to participate. After Institutional Review Board (Ethics Committee of Zhongshan Hospital) approval was obtained, the present study performed a retrospective review, which included all patients diagnosed with and treated for scalp angiosarcomas at Zhongshan Hospital, Fudan University (Shanghai, China), between January 2002 and December 2013. A total of 42 patients were included in the present study, 25 (60\%) were male and 17 (40\%) were female, and the patient age at the time of diagnosis age ranged between 19 and 82 years (median, 60 years). Written informed consent was obtained from all patients and/or family members. Cases with an unclear histological diagnosis and without a complete medical history were excluded from the present study. Patients with facial angiosarcoma, Stewart-Treves and angiosarcoma on trunk or extremities were also excluded.

Patient and disease characteristics. Epidemiological data including patient demographics, tumor characteristics (such 
as location, size and margin status), tumor grade, treatment parameters, and outcome (local recurrence and metastases) were obtained and analyzed. Tumor grade was stratified into two groups; low and high, as described previously (8). Among these patients, 25 (60\%) were male and 17 (40\%) were female, and the patient age at the time of diagnosis age ranged between 19 and 82 years (median, 60 years). Based on the type of lesion, patients were divided into a nodular localized lesion group (Fig. 1) and a diffuse lesion group (Fig. 2). The tumor presented as a nodular localized lesion in 25/42 patients $(60 \%)$ and as a diffuse lesion in $17 / 42$ patients (40\%). OS and recurrence-free survival (RFS) rates were listed.

Patients included in the present research provided their complete medical history, underwent a complete physical examination, routine laboratory tests and appropriate imaging examinations (computed tomography and magnetic resonance imaging) prior to undergoing treatment. Through a review of the pathology slides, a diagnosis of angiosarcoma was histologically confirmed in each case by two pathologists affiliated to Zhongshan Hospital (Fudan University) at the time of patient presentation.

Percentages were used to describe characteristics of patients, disease and treatment algorithms. OS and RFS rates were calculated and compared with those described in published literature (8). OS rate was calculated as the time from the date of diagnosis to the date of patient mortality. RFS rate was calculated as the time from date the patient was considered disease-free to the date of recurrence or mortality. Multimodality therapy was defined for treatment presenting $\geq 2$ modalities including surgery, radiation and chemotherapy.

Statistical analysis. Continuous variables were presented as mean \pm standard deviation or median with range and interquartile range depending on their distribution. Categorical variables were presented as frequency and percent. Kaplan-Meier analysis was used to calculate actuarial curves for OS and RFS rates, and a log-rank test to compare survival. A Cox proportional hazard model was used for multivariate analysis to test the predictive ability of clinical variables. The $\chi^{2}$ or Fisher exact test was used to test for differences between proportions for categorical variables. $\mathrm{P}<0.05$ was considered to indicate a statistically significant difference. Unless otherwise stated in the text, all P-values were two-sided. All statistical analyses were performed using SPSS 22.0 software (IBM Corp.).

\section{Results}

Patient and disease characteristics. The patient characteristics are presented in Table I. A total of 42 cases were clearly documented and served as the cohort for this analysis. In all patients, the primary lesions were located on the scalp. The tumor presented either as a nodular localized lesion in $25 / 42$ patients $(60 \%)$ or as a diffuse lesion in $17 / 42$ patients (40\%). The present study recorded characteristics such as gross tumor size. The maximal dimension ranged between 2 and $9 \mathrm{~cm}$, and the size was $\leq 5.0 \mathrm{~cm}$ in 19 patients $(45 \%)$ and $>5.0 \mathrm{~cm}$ in 23 patients $(55 \%)$, with a median size of $6.0 \mathrm{~cm}$. Patients did not exhibit any signs of distant metastatic disease outside the scalp at the time of diagnosis.
Treatment. Definitive surgical resection was the most common therapeutic measure, and was performed in 39 (93\%) patients, alone (12 patients), in combination with chemotherapy (14 patients), radiotherapy (6 patients) or 3-modality-therapy (7 patients). The remaining three patients received targeted therapy with apatinib. When treatment algorithms included surgery, chemotherapy and radiotherapy, the chemotherapy and radiotherapy were administered post-operatively. Surgical resection margins were at $>3 \mathrm{~cm}$, wherever possible, around any visible or palpable tumor. Graft or flap reconstruction was performed in patients who received surgery.

Combination of surgery, chemotherapy and radiotherapy was performed in 7 patients. Radiation therapy was delivered to the entire scalp post-operatively in $13(31 \%)$ patients. The median radiotherapy dose for patients treated with radiotherapy following surgery was $45 \mathrm{~Gy}$ (range, 40.0-60.0 Gy). A total of $21(50 \%)$ patients received chemotherapy as an adjuvant treatment. Adjuvant chemotherapy consisted of doxorubicin liposomes in 16 patients and cyclophosphamide alone in 5 patients. A total of $3(7 \%)$ patients received apatinib as the sole treatment. No severe complications associated with treatments were detected.

Patient outcomes. The median follow-up time of patients was 28.5 months (range, 5-107 months). A total of 37 (88\%) patients succumbed to mortality from angiosarcoma of the scalp. The 2-, 3- and 5-year OS rates were 64, 29 and 19\%, respectively (Fig. 3). A total of 23 (55\%) patients experienced tumor recurrence. The RFS rate was 55, 26 and $10 \%$ at 2, 3 and 5 years, respectively (Fig. 4). At 1- and 3-years following recurrence, the OS rate was 25 and $10 \%$, respectively (Fig. 5). Only two patients developed distant metastasis in the lungs.

Based on multivariate analysis, no treatment factors were significantly predictive of worse OS or RFS rates (data not shown). In the univariate analysis, patients aged $\geq 70$ years had a significantly worse $\mathrm{OS}$ rate $(\mathrm{P}=0.0071$; Fig. 6) and a worse RFS rate ( $\mathrm{P}=0.0095$; Fig. 7). Among tumor characteristics, only patients whose tumor presented as a nodular localized lesion had a significantly improved OS ( $\mathrm{P}=0.0079$; Fig. 8) and RFS ( $\mathrm{P}=0.0041$; Fig. 9) rates. Prognostic factors including tumor presentations, tumor size, tumor grade and treatment algorithms indicated that no factor was significantly predictive of tumor recurrence. In addition, no significant differences were observed between the responses of different tumor presentations to chemotherapy or radiation (Table II).

\section{Discussion}

Scalp angiosarcoma is a malignant aggressive head soft tissue neoplasm often with an innocuous presentation $(5,6)$. Despite ongoing researches, the treatment options for angiosarcoma are limited, and even following aggressive therapy, patient survival is very poor $(2,7)$. Therefore, timely and accurate diagnosis of this disease is critical in order to ensure an optimal patient outcome. However, initial diagnosis is often difficult due to a lack of definitive symptoms (8).

In the present study, the 5-year OS rate was $19 \%$, and the 5-year RFS rate was $10 \%$, with these demographic results being similar to previous reports (4,9-11). A total of $23(55 \%)$ patients experienced disease recurrence, and this result was 
Table I. Characteristics of 42 patients with scalp angiosarcoma treated at Zhongshan Hospital Fudan University.

\section{Characteristic}

Patients, n (\%)

\section{Sex}

Male

Female

Age, years

$<70$

$\geq 70$

Tumor size, $\mathrm{cm}$

$\leq 5$

$>5$

Tumor presentation

Nodular

Diffusion

Local therapy

Surgery alone

Radiation alone

Chemotherapy alone

Target therapy alone

Combine therapy

Surgery+chemotherapy

Surgery+radiation

Surgery+chemotherapy+radiation

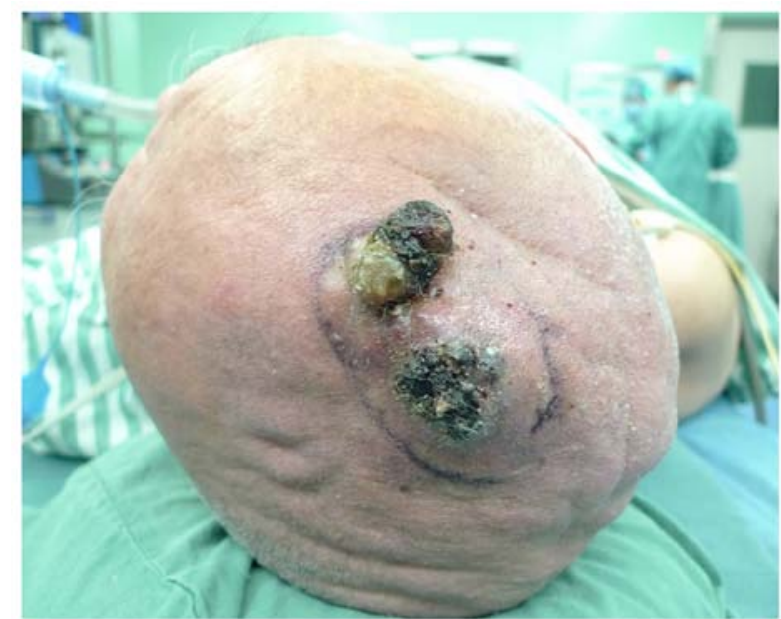

Figure 1. Scalp angiosarcoma presenting as a nodular localized lesion. Typical appearance of a nodular localized lesion. The marked area shows the visible edge of the mass.

consistent with a previously reported case (12). In our surgical practice, it was noted that patients with nodular tumor presentation usually had definitive local resection, while patients with diffuse presentation usually had uncertain margins that may be associated with recurrence. Other institutional reviews of angiosarcoma revealed that there was no significant difference in rates of loco-regional recurrence among the different tumor presentations or treatment modalities $(7,13)$.

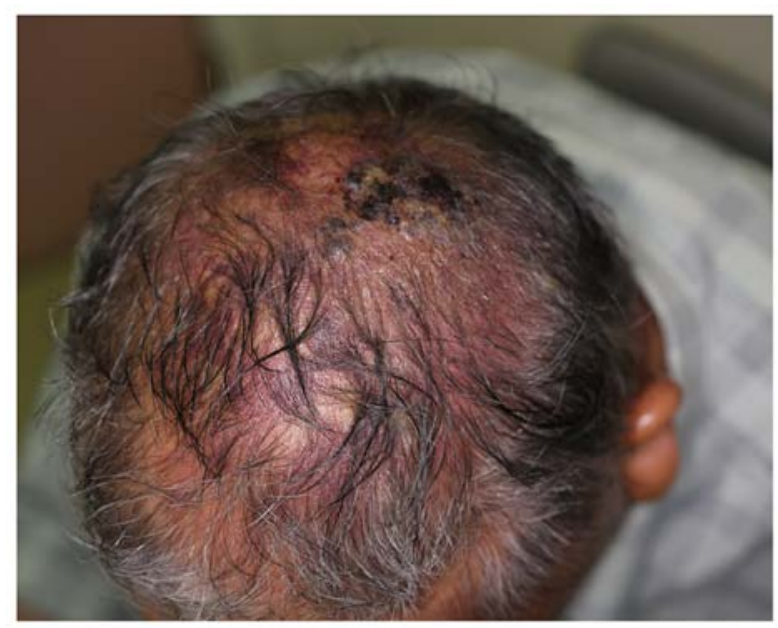

Figure 2. Scalp angiosarcoma presenting as a diffuse lesion.

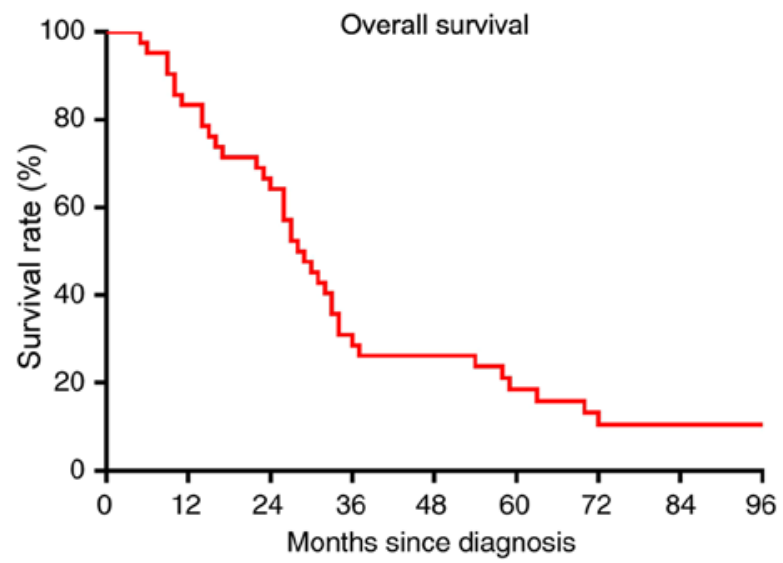

Figure 3. Overall survival rate of patients with scalp angiosarcoma. The 2-, 3 - and 5-year overall survival rates were 64, 29 and 19\%, respectively.

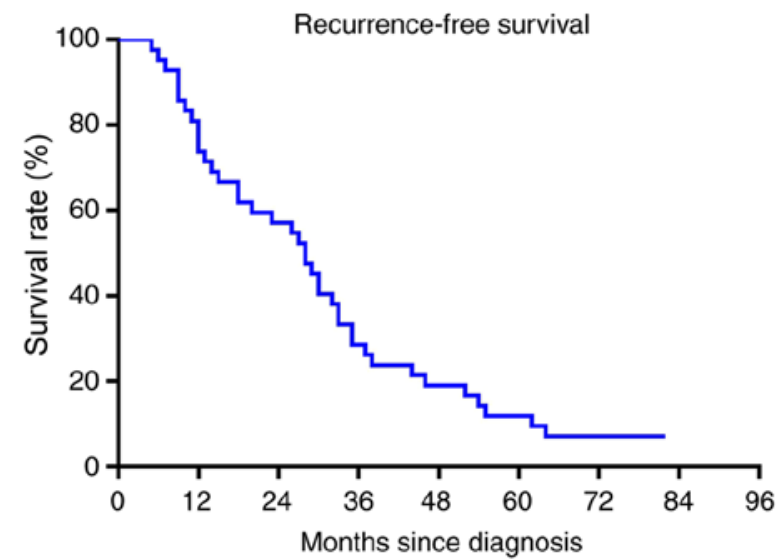

Figure 4. Recurrence-free survival rates of patients with scalp angiosarcoma. The recurrence-free survival rate was 55,26 and $10 \%$ at 2, 3 and 5 years, respectively.

Guadagnolo et al (9) reported that tumor size $>5 \mathrm{~cm}$ was significantly indicative of a worse prognosis for OS rate; however, the present study did not observe a statistically significant association between these two parameters. 


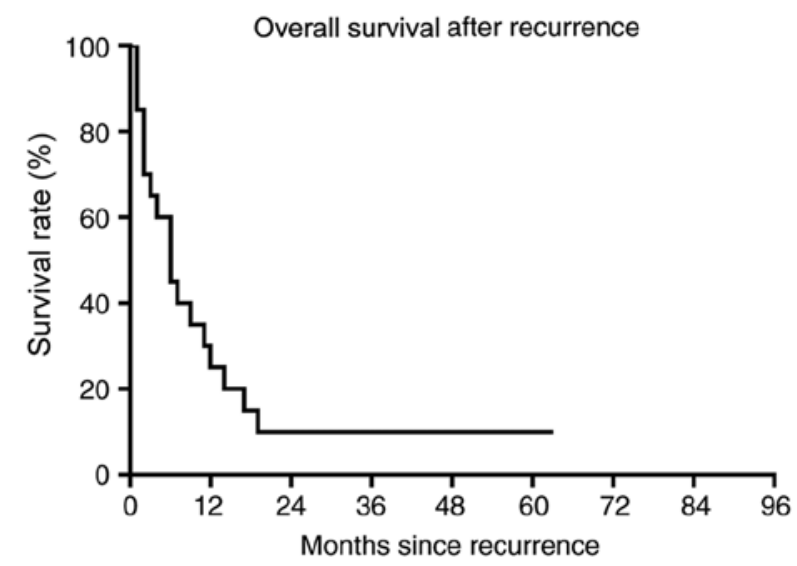

Figure 5. Overall survival after recurrence in patients with scalp angiosarcoma. At 1- and 3-years following recurrence, the overall survival rate was 25 and $10 \%$, respectively.

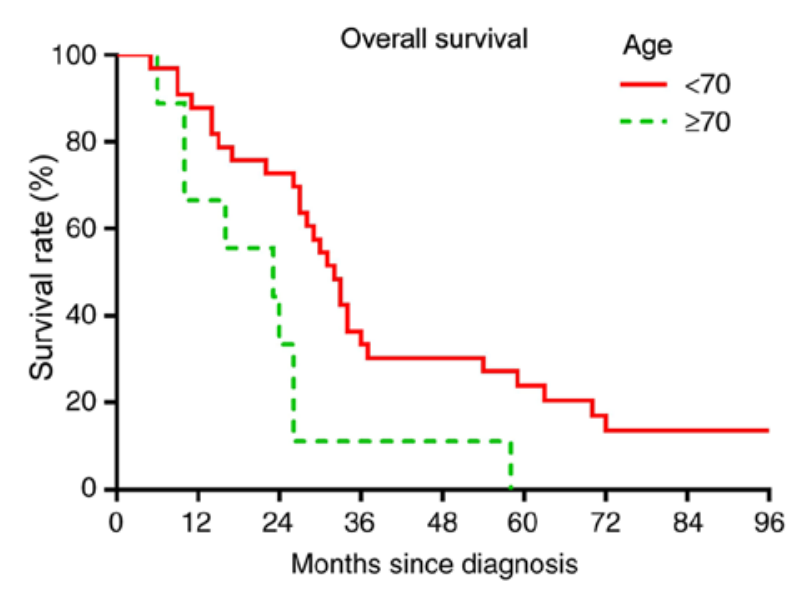

Figure 6. Overall survival comparison based on age. Log-rank test, $\mathrm{P}=0.0071$.

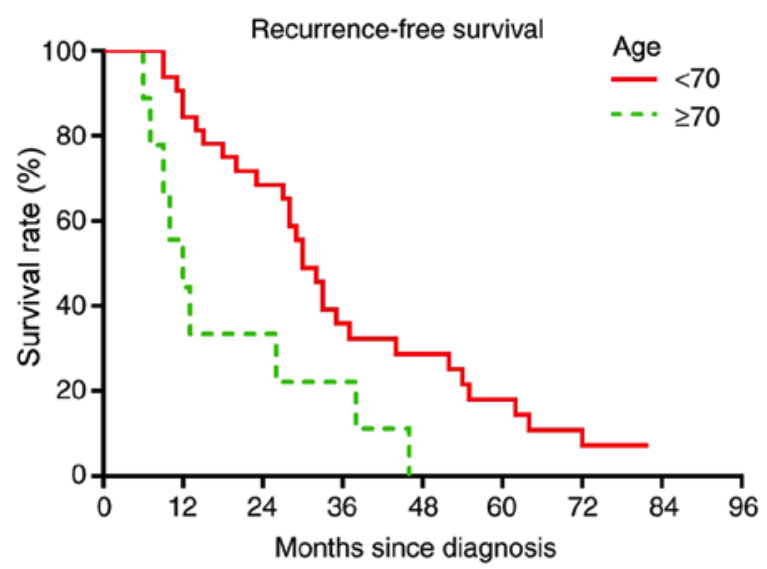

Figure 7. Recurrence-free survival comparison based on age. Log-rank test, $\mathrm{P}=0.0095$.

In the present study, only two (5\%) patients developed distant metastasis. This low distant metastatic rate was inconsistent with previously reported cases $(10,11)$. This inconsistency may be due to the fact that an autopsy was not performed in the majority of cases, therefore some metastases may have been neglected.

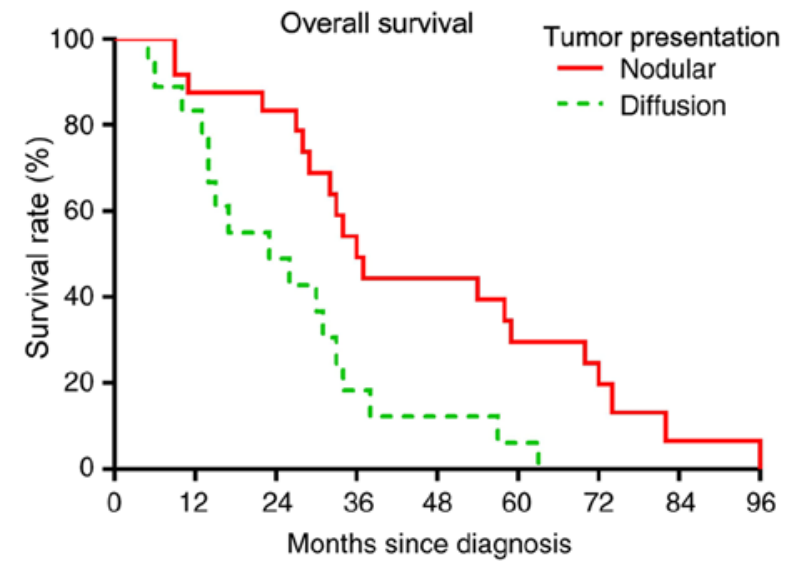

Figure 8. Overall survival comparison based on tumor presentation. Wilcoxon test, $\mathrm{P}=0.0078$.

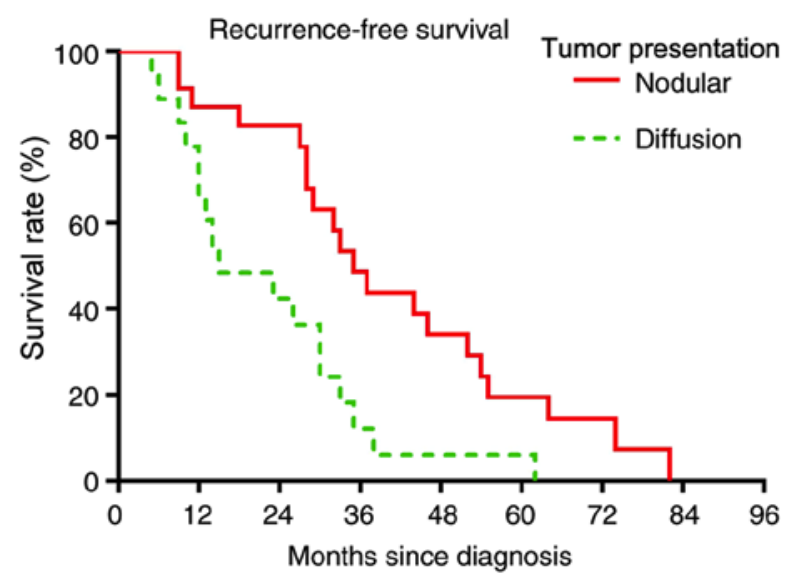

Figure 9. Recurrence-free survival comparison based on tumor presentation. Wilcoxon test, $\mathrm{P}=0.0058$.

It has been previously reported that angiosarcoma can be caused by therapeutic radiation or chronic lymphedema; therefore, secondary breast angiosarcomas are an important subgroup of angiosarcomas (14). However, in the present study, no patients presented with a history of radiotherapy or chronic lymphedema. In the department of Plastic and Reconstructive Surgery, Zhongshan Hospital (Shanghai, China), the most commonly observed presentation of angiosarcoma was on the scalp.

Morgan et al (15) demonstrated that a positive surgical margin was associated with poor OS in 44 patients with angiosarcoma of the face and scalp. However, in the current literature, the implication of surgical margins on the outcome of scalp angiosarcoma is still debated, and multiple studies did not identify such correlation $(4,7,9)$. In the present study, surgical resection margin for visible tumor was at $\geq 3 \mathrm{~cm}$. This extended resection aimed to obtain negative surgical margin status, which coincided with literature that supported the role of curative-intent surgery, irrespective of the histological surgical margin status (16). Reconstructive options involve skin grafting, local pedicled and axial flaps, and microsurgery for complex and extensive wound reconstruction (13). If bone invasion was not present, the skull periosteum and graft skin were retained (12). 
Table II. Univariate analysis of overall survival rate and recurrence-free survival rate in 42 patients with scalp angiosarcoma treated at Zhongshan Hospital Fudan University.

\begin{tabular}{|c|c|c|c|c|c|}
\hline Variable & Patients, n (\%) & 5-year OS, \% & P-value & 5-year RFS, \% & P-value \\
\hline Sex & & & 0.177 & & 0.092 \\
\hline Male & $25(60)$ & 23 & & 18 & \\
\hline Female & $17(40)$ & 11 & & 0 & \\
\hline Age, years & & & 0.007 & & \\
\hline$<70$ & $33(79)$ & 23 & & 15 & $0.010^{\mathrm{a}}$ \\
\hline$\geq 70$ & $9(21)$ & 0 & & 0 & \\
\hline Tumor presentation & & & 0.003 & & $0.004^{\mathrm{a}}$ \\
\hline Nodular & $24(57)$ & 29 & & 19 & \\
\hline Diffusion & $18(43)$ & 6 & & 0 & \\
\hline Tumor grade & & & 0.936 & & 0.987 \\
\hline Low & $12(29)$ & 17 & & 13 & \\
\hline High & $30(71)$ & 15 & & 15 & \\
\hline Tumor size, $\mathrm{cm}$ & & & 0.979 & & 0.961 \\
\hline$\leq 5$ & $19(45)$ & 16 & & 12 & \\
\hline$<5$ & $23(55)$ & 15 & & 15 & \\
\hline Local therapy & & & 0.517 & & 0.683 \\
\hline $\mathrm{S}$ & $12(29)$ & 25 & & 25 & \\
\hline $\mathrm{S}+\mathrm{RT}$ & $6(14)$ & 0 & & 0 & \\
\hline Multimodality therapy & & & 0.587 & & 0.628 \\
\hline $\mathrm{S}+\mathrm{C}$ & $14(33)$ & 14 & & 7 & \\
\hline $\mathrm{S}+\mathrm{RT}$ & $6(14)$ & 0 & & 0 & \\
\hline $\mathrm{S}+\mathrm{RT}+\mathrm{C}$ & 7 (17) & 15 & & 0 & \\
\hline
\end{tabular}

${ }^{\mathrm{a} P}<0.05$. OS, overall survival; RFS, recurrence-free survival; S, surgery; RT, radiation therapy; C, chemotherapy.

Current treatment for angiosarcomas of the scalp, face and neck typically involves surgery and neoadjuvant/adjuvant chemotherapy $(4,7)$. In this study, it was noted that no patients survived without chemotherapy treatment, suggesting that chemotherapy played an important part in angiosarcoma research. The study by Chow et al (17) reported that monotherapy with the $\beta$-blocker propranolol was capable of reducing the proliferative index of a cutaneous angiosarcoma. In the study conducted by Singla et al (18), patients received paclitaxel-based regimens. Similarly, Schlemmer et al (19) reported that patients with advanced angiosarcoma who were treated with paclitaxel had a significantly improved survival. However, no assumptions can be made pertaining to the OS from these studies. Gebhardt et al (20) reported that complete remission of treatment-refractory advanced scalp angiosarcoma occurred following protracted intralesional interleukin-2 therapy. Young et al (21) investigated vascular-targeted agents, including bevacizumab [an anti-vascular endothelial growth factor (VEGF) antibody], axitinib [a VEGF receptor (VEGFR) tyrosine kinase inhibitor], everolimus (an mTOR inhibitor), selumetinib (a mitogen-activated protein kinase kinase inhibitor) and vadimezan (a vascular-disrupting agent) on cutaneous angiosarcoma cell lines, showing positive responses. However, a subsequent clinical study indicated that patients did not benefit from VEGF/VEGFR targeted treatment (22). Additional studies with expanded patient populations are necessary to fully examine the adjuvant natures of these agents in the setting of other therapeutic regimens. In the present study, three patients received apatinib, a tyrosine kinase inhibitor that selectively inhibits VEGFR2; however, it did not significantly improve the outcome of the patients.

The complete pathogenesis of angiosarcoma remains unknown, and it is still a matter of debate whether angiosarcomas are derived from blood or lymph vessels or their progenitors (23). In recent years, multiple studies have sought to establish the molecular pathogeneses of angiosarcomas and identify specific targets for its treatment: Wada et al (24) investigated the effect of inhibitors of the PI3K/AKT/mTOR pathway using ISOS-1 and ISO-HAS angiosarcoma cell lines, and demonstrated that the phosphoinositide-dependent kinase 1 (PDK1) inhibitor was a promising therapeutic agent. In addition, Tsuneki et al (25) identified an agent named survivin as a promising biomarker and critical regulator of cellular proliferation for angiosarcoma using human angiosarcoma specimens, and also suggested that YM155 may function as a potential therapeutic agent.

However, there are limitations to the present study, as it was retrospective regarding data collection, and could be influenced by the inherent bias of study design. The present study spanned over a period of 12 years, and has been accompanied by rapid 
advances in the treatment modalities. Therefore, selection bias may be involved due to the heterogeneous treatment. In conclusion, the present study indicates that scalp angiosarcoma is an aggressive malignancy with a high mortality rate and poor prognosis. Despite the fact that the exact mechanisms underlying angiosarcoma remain unclear, the research on cutaneous angiosarcoma genomics is preliminary but consistent (26).

\section{Acknowledgements}

Not applicable

\section{Funding}

The present study was supported from the National Natural Science Foundation of China (grant no. 81802724).

\section{Availability of data and materials}

All data generated or analyzed during this study are included in this published article.

\section{Authors' contributions}

JL, YY, MZ, CC, NL, FQ and YZ designed and performed the experiments, analyzed the data and wrote the paper. All authors read and approved the final version of the manuscript.

\section{Ethics approval and consent to participate}

The study was approved by the Ethics Committee of Zhongshan Hospital, Shanghai, China. Written informed consent was obtained from all patients and/or family members.

\section{Patient consent for publication}

All patients provided written informed consent for the publication of the present study and images.

\section{Competing interests}

All authors declare that they have no competing interests.

\section{References}

1. Wanebo HJ, Koness RJ, MacFarlane JK, Eilber FR, Byers RM, Elias EG and Spiro RH: Head and neck sarcoma: Report of the Head and Neck Sarcoma Registry. Society of Head and Neck Surgeons Committee on Research. Head Neck 14: 1-7, 1992.

2. Bernstein JM, Irish JC, Brown DH, Goldstein D, Chung P, Razak A, Catton C, Gilbert RW, Gullane PJ and O'Sullivan B: Survival outcomes for cutaneous angiosarcoma of the scalp versus face. Head Neck 39: 1205-1211, 2017.

3. Mendenhall WM, Mendenhall CM, Werning JW, Reith JD and Mendenhall NP: Cutaneous angiosarcoma. Am J Clin Oncol 29: 524-528, 2006

4. Pawlik TM, Paulino AF, McGinn CJ, Baker LH, Cohen DS, Morris JS, Rees R and Sondak VK: Cutaneous angiosarcoma of the scalp: A multidisciplinary approach. Cancer 98: 1716-1726, 2003.

5. Ye J, Li XF, Wang YD and Yuan Y: Long-term survival of a patient with scalp angiosarcoma and multiple metastases treated using combination therapy: A case report. Oncol Lett 9: $1725-1728,2015$

6. Lin SC and Chang TS: Cutaneous angiosarcoma of the scalp mimicking facial cellulitis. Ear Nose Throat J 95: 438-440, 2016.
7. Fury MG, Antonescu CR, Van Zee KJ, Brennan MF and Maki RG: A 14-year retrospective review of angiosarcoma: Clinical characteristics, prognostic factors, and treatment outcomes with surgery and chemotherapy. Cancer J 11: 241-247, 2005.

8. Perez MC, Padhya TA, Messina JL, Jackson RS, Gonzalez RJ Bui MM, Letson GD, Cruse CW, Lavey RS, Cheong D, et al: Cutaneous angiosarcoma: A Single-Institution experience. Ann Surg Oncol 20: 3391-3397, 2013.

9. Guadagnolo BA, Zagars GK, Araujo D, Ravi V, Shellenberger TD and Sturgis EM: Outcomes after definitive treatment for cutaneous angiosarcoma of the face and scalp. Head Neck 33: 661-667, 2011.

10. Ogawa K, Takahashi K, Asato Y, Yamamoto Y, Taira K, Matori S, Iraha S, Yagi N, Yogi A, Haranaga S, et al: Treatment and prognosis of angiosarcoma of the scalp and face: A retrospective analysis of 48 patients. Br J Radiol 85: e1127-e1133, 2012.

11. Patel SH, Hayden RE, Hinni ML, Wong WW, Foote RL, Milani S, Wu Q, Ko SJ and Halyard MY: Angiosarcoma of the scalp and face: The Mayo clinic experience. JAMA Otolaryngol Head Neck Surg 141: 335-340, 2015.

12. Lee BL, Chen CF, Chen PC, Lee HC, Liao WC, Perng CK, Ma H and $\mathrm{Lin} \mathrm{CH}$ : Investigation of prognostic features in primary cutaneous and soft tissue angiosarcoma after surgical resection: A retrospective study. Ann Plast Surg 78 (Suppl 2): S41-S46, 2017.

13. Abraham JA, Hornicek FJ, Kaufman AM, Harmon DC, Springfield DS, Raskin KA, Mankin HJ, Kirsch DG, Rosenberg AE, Nielsen GP, et al: Treatment and outcome of 82 patients with angiosarcoma. Ann Surg Oncol 14: 1953-1967, 2007.

14. Young RJ, Brown NJ, Reed MW, Hughes D and Woll PJ: Angiosarcoma. Lancet Oncol 11: 983-9891, 2010.

15. Morgan MB, Swann M, Somach S, Eng W and Smoller B: Cutaneous angiosarcoma: A case series with prognostic correlation. J Am Acad Dermatol 50: 867-874, 2004.

16. Oashi K, Namikawa K, Tsutsumida A, Takahashi A, Itami J, Igaki $\mathrm{H}$, Inaba $\mathrm{K}$ and Yamazaki N: Surgery with curative intent is associated with prolonged survival in patients with cutaneous angiosarcoma of the scalp and face - a retrospective study of 38 untreated cases in the Japanese population. Eur J Surg Oncol 44: 823-829, 2018.

17. Chow W, Amaya CN, Rains S, Chow M, Dickerson EB and Bryan BA: Growth attenuation of cutaneous angiosarcoma with propranolol-mediated $\beta$-blockade. JAMA Dermatol 151: 1226-1229, 2015.

18. Singla S, Papavasiliou P, Powers B, Gaughan J, von Mehren M, Watson JC and Farma JM: Challenges in the treatment of angiosarcoma: A single institution experience. Am J Surg 208: 254-259, 2014

19. Schlemmer M, Reichardt P, Verweij J, Hartmann JT, Judson I, Thyss A, Hogendoorn PC, Marreaud S, Van Glabbeke M and Blay JY: Paclitaxel in patients with advanced angiosarcomas of soft tissue: A retrospective study of the EORTC soft tissue and bone sarcoma group. Eur J Cancer 44: 2433-2436, 2008.

20. Gebhardt C, Ziegler B, Stadler S, Goerdt S and Utikal J: Complete remission of treatment-refractory advanced angiosarcoma of the scalp by protracted intralesional interleukin-2 therapy. Br J Dermatol 172: 1156-1158, 2015.

21. Young RJ, Woll PJ, Staton CA, Reed MW and Brown NJ: Vascular-targeted agents for the treatment of angiosarcoma. Cancer Chemother Pharmacol 73: 259-270, 2014.

22. Young RJ and Woll PJ: Anti-angiogenic therapies for the treatment of angiosarcoma: A clinical update. Memo 10: 190-193, 2017.

23. Donghi D, Kerl K, Dummer R, Schoenewolf N and Cozzio A: Cutaneous angiosarcoma: Own experience over 13 years. Clinical features, disease course and immunohistochemical profile. J Eur Acad Dermatol 24: 1230-1234, 2010.

24. Wada M, Horinaka M, Yasuda S, Masuzawa M, Sakai T and Katoh N: PDK1 is a potential therapeutic target against angiosarcoma cells. J DermatoL Sci 78: 44-50, 2015.

25. Tsuneki M, Kinjo T, Mori T, Yoshida A, Kuyama K, Ohira A, Miyagi T, Takahashi K, Kawai A, Chuman H, et al: Survivin: A novel marker and potential therapeutic target for human angiosarcoma. Cancer Sci 108: 2295-2305, 2017.

26. Shustef E, Kazlouskaya V, Prieto VG, Ivan D and Aung PP: Cutaneous angiosarcoma: A current update. J Clin Pathol 70: 917-925, 2017.

This work is licensed under a Creative Commons Attribution-NonCommercial-NoDerivatives 4.0 International (CC BY-NC-ND 4.0) License. 